\title{
Observations of unusual whistlers during daytime at Jammu
}

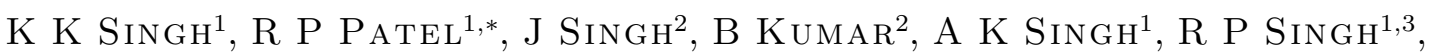 \\ B L KOUL ${ }^{4}$ and LALMANI ${ }^{4}$ \\ ${ }^{1}$ Atmospheric Research Laboratory, Physics Department, Banaras Hindu University, Varanasi, India. \\ ${ }^{2}$ Physics Department, G. G. M. Science College, Canal Road, Jammu, India. \\ ${ }^{3}$ Vice-Chancellor, V. K. S. University, Arrah, Bihar, India. \\ ${ }^{4}$ Physics Department, National Institute of Technology Srinagar, Camp Classes, \\ Old University Campus, Canal Road, Jammu, India. \\ *e-mail: rppatel12@yahoo.co.in_abhay_s@rediffmail.com
}

In this paper, we report observations of unusual whistlers recorded at Jammu (geomag. lat. = $22^{\circ} 26^{\prime} \mathrm{N} ; L=1.17$ ), India on March 8, 1999 during the daytime. They are interpreted as one-hop ducted whistlers having propagated along higher $L$-values in closely spaced narrow ducts from the opposite hemispheres. After leakage from the duct, the waves might have propagated in the earthionosphere waveguide towards the equator in surface mode. Tentative explanation of the dynamic spectra of these events is briefly presented.

\section{Introduction}

Whistler studies in India have made significant contributions to the propagation of low latitude whistlers and the determination of various plasma parameters in the inner plasmasphere/ magnetoshpere (Somayajulu et al 1972; Singh 1993; Singh et al 1998; Singh and Hayakawa 2001; Singh et al 2004a, 2006). Although the propagation mechanisms of night-time whistlers are known to some extent, the same cannot be said for daytime whistlers because of the scarcity of the daytime whistler data. Daytime whistlers in small numbers were reported from the Japanese stations (Hayakawa and Tanaka 1978). Due to high absorptions during daytime, whistlers at low latitude from Indian stations are rarely observed. Under All India Coordinated Program of Ionosphere Thermosphere Studies (AICPITS), continuous observation of whistlers and VLF emissions both during day and night hours at Jammu was carried out. We have found some interesting events of whistlers during daytime, which are diffused and appear to be doublets and triplets. Whereas whistler triplets have been reported from low latitude station Agra (geomag. lat., $17^{\circ} 1^{\prime} \mathrm{N} ; L=1.15$ ) during night-time (Singh et al 1997). To the knowledge of the authors, there is no report of the whistler doublets and triplets observed during the daytimes at low latitudes, although observations from high latitudes exist (Helliwell 1965). Whistler doublets have been reported from ISIS2 satellite (Thomson 1977) and ACTIVE satellite (Lichtenberger et al 1991). Chum et al (2006) analyzed whistlers observed on the DEMETER and MAGION-5 satellite and lightning discharges by EUCLID and demonstrated that the area in the ionosphere through which the electromagnetic energy induced by a lightning discharge enters into the magnetosphere as whistler mode waves is up to several thousands of kilometers wide. They have also demonstrated that positive and negative cloud to ground discharges has approximately the same efficiency in producing whistlers. Recently, Ferencz et al (2007) have analyzed VLF data of DEMETER satellite and reported signal structure exhibiting

Keywords. Unusual whistlers; ducted propagation; waveguide mode; one hop. 
numerous fractional - hop whistlers. The structure has been explained by considering that the lightning generated electromagnetic energy first propagates through the Earth-ionosphere waveguide and then through the ionosphere and magnetosphere. In this paper we report observations of doublets and triplets recorded during daytime for the first time at a low latitude ground station Jammu.

\section{Experimental results}

The experimental set-up employed at our low latitude ground station Jammu to record the VLF signals consists of a T-type antenna, pre- and mainamplifiers having a bandwidth of $50-15 \mathrm{kHz}$ and a magnetic tape recorder. T-type antenna with 25 meter vertical length, 6 meter horizontal length and $3.2 \mathrm{~mm}$ diameter (having impedance about $1 \mathrm{M} \Omega$ ) has been used to record vertical component of wave electric field. The antenna is rendered aperiodic with the help of a suitable RC network, to avoid any possible ringing effect (Singh et al 2004b). The whistler voltage induced in the antenna is amplified and recorded on magnetic tape recorder. The gain of the pre/main amplifier is varied manually from 0 to $40 \mathrm{~dB}$, to avoid overloading of the amplifier at the time of intense whistler activity. We did not have automatic recording set-up. Further, automatic recording system will not be very much useful due to very low occurrence rate. The observations were taken continuously both during the day and night-times on a routine basis. The data were stored on magnetic tapes and then analyzed using a digital sonograph. During analysis digitization of the analog signal is carried out at $16 \mathrm{kHz}$ sampling frequency. The inbuilt software in the spectrum analyzer provides dynamic spectra, which update in real time typically covering $8 \mathrm{kHz}$ in frequency and 2.54 seconds in time. The frequency range of the spectrum analyzer could vary between $100 \mathrm{~Hz}$ and $40 \mathrm{kHz}$. The results of the analysis showed a number of whistlers and VLF emissions. A large number of events of VLF waves have been recorded, analyzed and published (Singh et al 2000; Singh et al 2004b; Singh et al 2007) during the span of seven years of observation. In this paper some unusual events such as the whistler doublets and the triplets observed during the daytime on March 8, 1999 are described.

Typical examples of daytime whistler doublet and triplet recorded on March 8, 1999 during disturbed magnetic activity period $\left(\mathrm{K}_{\mathrm{P}}=20_{-}\right)$are presented in figures 1 and 2. The whistler activity started at 1435 IST (Indian Standard Time) and continued for more than an hour. Figure 1 shows the dynamic spectrum of two whistler traces separated by about $0.16 \mathrm{~s}$, which are referred to as

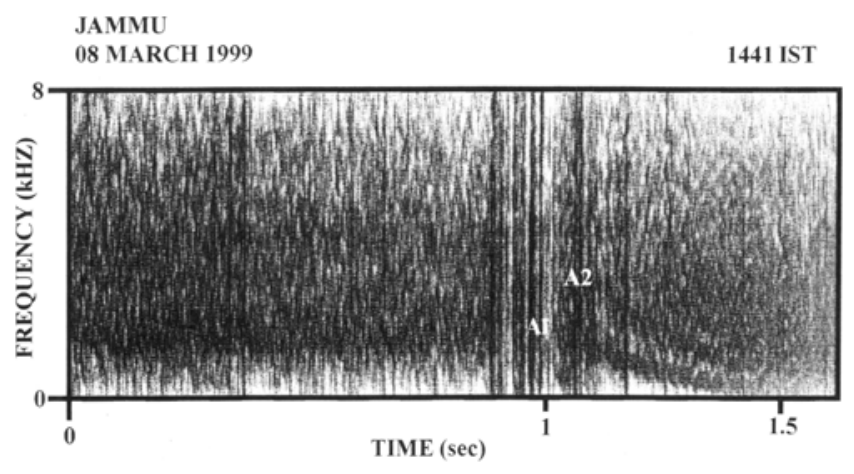

Figure 1. Spectrogram of whistler doublet observed during daytime at Jammu (geomag. lat. $=22^{\circ} 26^{\prime} \mathrm{N} ; L=1.17$ ) on 8 March 1999 at 1441 IST.

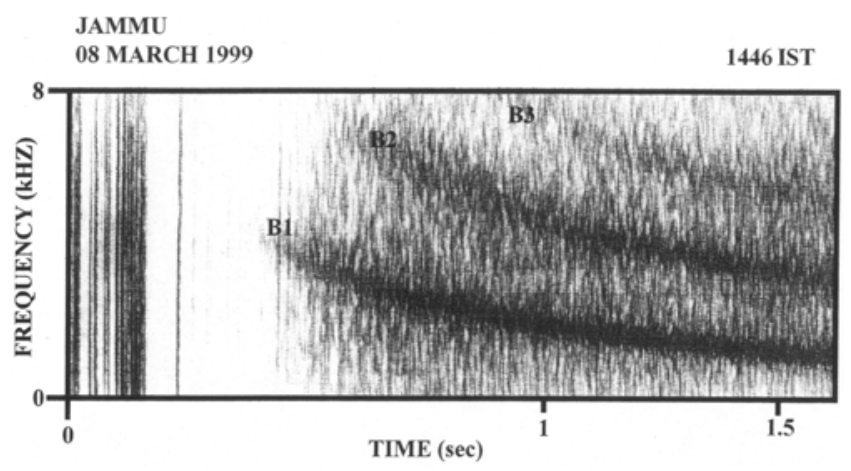

Figure 2. Spectrogram of whistler triplet observed during daytime at Jammu (geomag. lat. $=22^{\circ} 26^{\prime} \mathrm{N} ; L=1.17$ ) on 8 March 1999 at 1446 IST.

whistler doublet recorded at 1441 IST in the frequency range of about $200 \mathrm{~Hz}$ to $2 \mathrm{kHz}$. The first trace (A1) of the doublet has the lower cut-off frequency $\sim 200 \mathrm{~Hz}$, and the upper cut-off frequency $\sim 1 \mathrm{kHz}$, whereas the second trace $(\mathrm{A} 2)$ has the lower cut-off frequency $\sim 500 \mathrm{~Hz}$ and upper cut-off frequency $\sim 2 \mathrm{kHz}$. The observed diffused whistler traces of the doublet indicate that they might have propagated through the ducts having diffused boundary (Singh 1993). Normal singlet whistlers recorded simultaneously along with whistler doublet and triplet on the same day (March 8, 1999) and during the same period are shown in figures 3 and 4 . The normal singlet whistler shown in figure 3 was recorded at 1438 IST, just before the observation of doublet and triplet whereas figure 4 shows the normal singlet whistler recorded at 1525 IST, just after the observed events. It is to be noted that just before the observation of whistler doublet, radio noise is intense in the whole frequency band, but it is not difficult to visualize the whistler signal. We have checked for the possibility of local artifact both from the instrument and the local noise and these are ruled out. Both the whistler doublet and associated noise are of natural origin. 


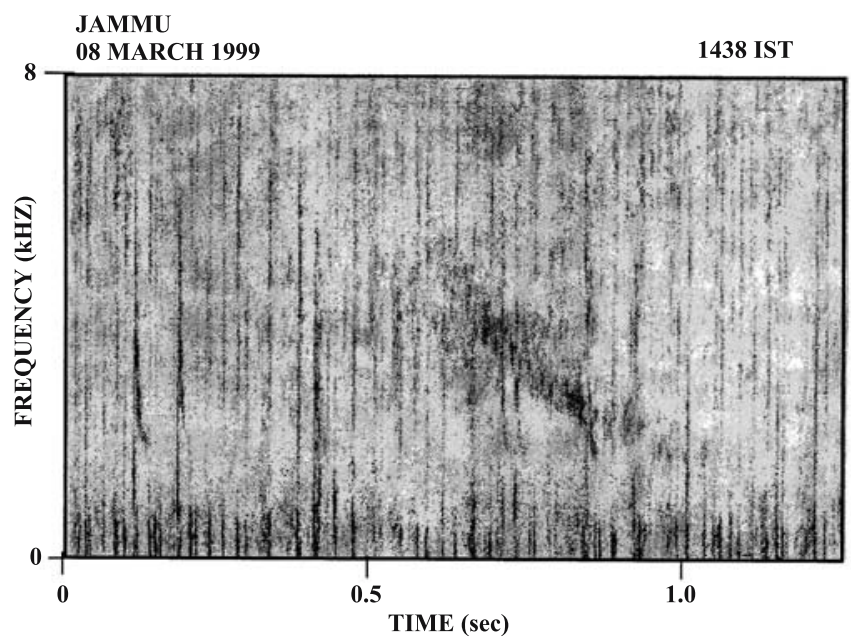

Figure 3. Spectrogram of whistler singlet observed during daytime at Jammu (geomag. lat. $=22^{\circ} 26^{\prime} \mathrm{N} ; L=1.17$ ) on 8 March 1999 at 1438 IST.

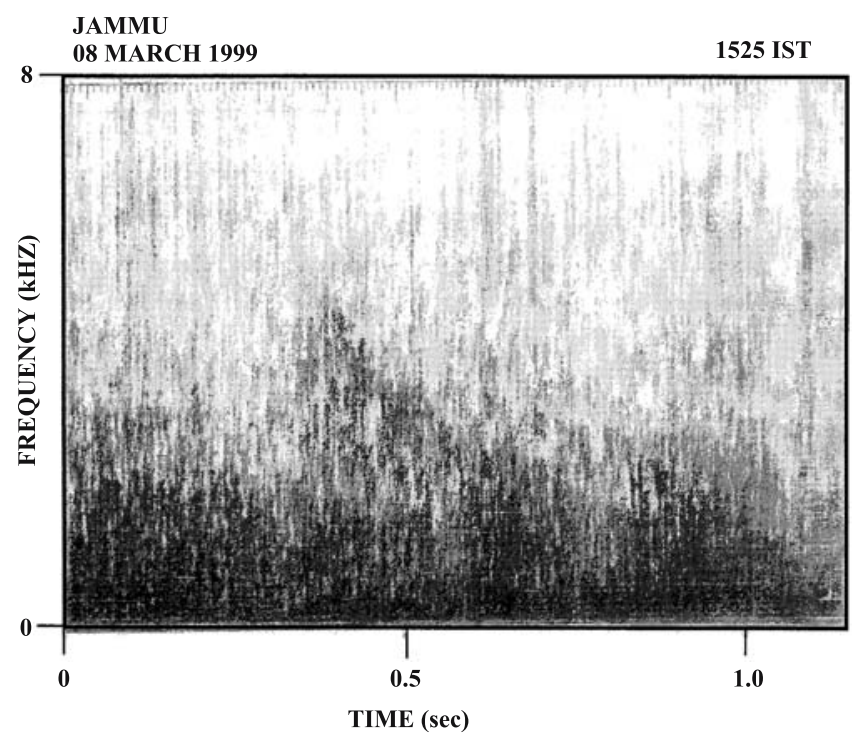

Figure 4. Spectrogram of whistler singlet observed during daytime at Jammu (geomag. lat. $=22^{\circ} 26^{\prime} \mathrm{N} ; L=1.17$ ) on 8 March 1999 at 1525 IST.

Figure 2 shows a typical example of whistler triplet, composed of three consecutive whistler traces separated by about $0.64 \mathrm{~s}$ and recorded at 1446 IST. The lower and the upper cut-off frequencies of the first whistler (B1) is about $1 \mathrm{kHz}$ and $3.5 \mathrm{kHz}$ respectively. The same for the second whistler (B2) and the third whistler (B3) is about 3.0 and $6.0 \mathrm{kHz}$ and $5.0 \mathrm{kHz}$ and $7.5 \mathrm{kHz}$ respectively. All the three whistler traces of the triplet are diffused and of longer periods and higher dispersion.

Individual elements of whistler doublet and triplet are analyzed using the curve fitting technique (Tarcsai 1975). The technique used in the present paper for the analysis of whistlers recorded at Jammu and the derivation of magnetospheric parameters is based on the determination of the parameters in Bernard's (1973) approximation and of a further parameter locating the causative sferic by a least square curve fitting procedure. In the following, an outline of the Tarcsai (1975) method used for the analysis of non-nose whistlers with known causative sferic observed at Jammu is described. The whistlers are known to propagate along geomagnetic field lines in ducted mode. The dispersion function under suitable approximation is written as (Bernard 1973)

$$
D(f)=t(f) \sqrt{f}=D_{0} \frac{\left(f_{H e}-A f\right)}{\left(f_{H e}-f\right)},
$$

where $D_{0}$ is zero-frequency dispersion, $f_{\mathrm{He}}$ is equatorial electron gyrofrequency, $t(f)$ travel time at frequency $f$, and

$$
A=\frac{3 \Lambda_{n}-1}{\Lambda_{n}\left(1+\Lambda_{n}\right)}, \quad \Lambda_{n}=\frac{f_{n}}{f_{H e}}
$$

$f_{n}$ is the nose frequency for which travel time $t_{n}$ is written as

$$
t_{n}=\frac{D_{0}}{\sqrt{f_{n}}} \frac{2}{\left(1+\Lambda_{n}\right)}
$$

Sometimes the causative sferics are not known. In such cases the travel time is measured from the chosen origin and a correction parameter $T$ is introduced (which gives the time difference between the chosen origin and the actual sferic). Using equations (1) and (2), the measured travel time $t^{*}(f)$ is written as

$$
t^{*}(f)=t(f)-T=\frac{D_{0}}{\sqrt{f}} \frac{f_{H e}-A f}{f_{H e}-f}-T .
$$

In this equation there are four unknown parameters $D_{0}, f_{H e}, T$, and $f_{n}$. Tarcsai (1975) has developed a computer programme to solve equation (4) for the unknown using successive iteration method. In this method those values of $D_{0}, f_{H e}, T$ and $f_{n}$ are searched which give best fit to the measured parameters. After Park (1972) and using equation (3) for $t_{n}$, the expressions used for computing the equatorial electron density $n_{\text {eq }}$ and total electron tube content $N_{T}$ are written as:

$$
t_{n}=8.736 \times 10^{5} \times f_{H e}^{-1 / 3},
$$


Table 1. Results of whistler curve fitting. (Date of whistler recording March 8, 1999).

\begin{tabular}{|c|c|c|c|c|c|c|c|}
\hline Whistler & $\begin{array}{c}\text { Time of } \\
\text { observation } \\
\text { hours } \\
\text { (IST) }\end{array}$ & Trace & $\begin{array}{c}\text { Dispersion } \\
D_{o}\left(\mathrm{~s}^{1 / 2}\right)\end{array}$ & $L$-value & $\begin{array}{c}\text { Nose } \\
\text { frequency } \\
f_{n}(\mathrm{kHz})\end{array}$ & $\begin{array}{c}\text { Equatorial } \\
\text { electron } \\
\text { density } \\
n_{\text {eq }}\left(\mathrm{cm}^{-3}\right)\end{array}$ & $\begin{array}{c}\text { Total } \\
\text { electron } \\
\text { content } N_{T} \\
10^{13} \mathrm{el} /\left(\mathrm{cm}^{2}\right. \\
\text { tube })\end{array}$ \\
\hline \multirow[t]{2}{*}{ Doublet } & 1441 & A1 & $16.8 \pm 1.1$ & $2.63 \pm 0.0$ & $17.59 \pm 6.28$ & $119 \pm 61$ & $0.02 \pm 0.01$ \\
\hline & & $\mathrm{A} 2$ & $18.6 \pm 0.6$ & $2.67 \pm 0.0$ & $16.86 \pm 3.02$ & $136 \pm 33$ & $0.24 \pm 0.004$ \\
\hline \multirow[t]{3}{*}{ Triplet } & 1446 & B1 & $81.9 \pm 1.1$ & $4.35 \pm 0.01$ & $9.93 \pm 0.01$ & $220 \pm 5$ & $2.91 \pm 0.08$ \\
\hline & & $\mathrm{B} 2$ & $95.2 \pm 1.4$ & $4.39 \pm 0.01$ & $9.82 \pm 0.02$ & $247 \pm 8$ & $3.39 \pm 0.13$ \\
\hline & & B3 & $100.2 \pm 1.8$ & $4.43 \pm 0.02$ & $9.81 \pm 0.02$ & $296 \pm 9$ & $3.78 \pm 0.12$ \\
\hline
\end{tabular}

where $f_{H e}$ is in $\mathrm{Hz}$,

$$
\begin{aligned}
& n_{\mathrm{eq}}=K_{e} f_{n} t_{n}^{2} L^{-5}=K_{e}^{\prime} D_{0}^{2} f_{H e}^{5 / 3}, \\
& N_{T}=K_{T} f_{n} t_{n}^{2} L^{-1}=K_{T}^{\prime} D_{0}^{2} f_{H e}^{1 / 3},
\end{aligned}
$$

where the constants $K_{e}^{\prime}$ and $K_{T}^{\prime}$ are weakly dependent on $f_{n}$ and $f_{\mathrm{He}}$.

This technique has been successfully used to analyze the night-time whistlers observed at low latitude Indian ground stations (Singh et al 1997, 1998, 2004a, 2006; Singh and Hayakawa 2001). The dynamic spectrum is processed to derive source time $T_{0}$, zero frequency dispersion $D_{0}$, nose frequency $f_{n}$ and equatorial electron gyrofrequency $f_{\text {Heq }}$ (Hamar et al 1990; Singh et al 1999). The derived whistler and magnetospheric parameters are given in table 1 . Here it should be noted that the upper cut of frequency of doublets are mixed with intense radio noise and hence these cannot be determined precisely. However, this does not affect the derived parameters because in the construction of theoretical whistlers the role of the upper/the lower cut of frequency is minimal. The diffuseness of the trace may cause little error in the measurement of arrival time and frequency. This is minimized by using digital technique and enlarging the dynamic spectra.

\section{Discussion}

The dynamic spectra shown in figures 1 and 2 are the first observation of daytime whistler doublet composed of two traces separated by about $0.16 \mathrm{~s}$ and, whistler triplet composed of three traces separated by about $0.64 \mathrm{~s}$ respectively. As these waves were observed at the ground station, they might have propagated in either ducted or prolongitudinal mode along the geomagnetic field lines. Whistler doublets observed by SAS equipment on board satellite ISIS 2 were interpreted in terms of prolongitudinal (PL) propagation (Thomson 1977), whereas whistler doublets observed in ACTIVE satellite have been interpreted in terms of ducted mode of propagation (Lichtenberger et al 1991). Singh et al (1997) have shown that whistler triplets recorded at Agra during night hours are the one hop multipath whistlers, which propagated to the ground station under the influence of equatorial anomaly. The time separation between the successive triplets was explained in terms of time intervals between the causative lightning discharges.

The dynamic spectra of whistler doublet and triplet as shown in figures 1 and 2 distinguishes it from the falling tone multiphase periodic VLF emissions usually observed at high latitudes, because the time separations between the consecutive whistler traces in doublets and triplets $(\sim 0.16-0.64 \mathrm{~s})$ are much smaller as compared to 2-6 s generally observed between the periodic emissions (Helliwell 1965). The dispersion analysis (table 1) shows that the recorded signals are not the first, the third, and the fifth hop whistlers generated from a stroke of a lightning discharge. An increase in dispersion shows that these signals may have propagated along field lines, which were closely spaced. The dispersion of whistlers $\mathrm{A} 1$ and $\mathrm{A} 2$ of the doublet is $16.8 \mathrm{~s}^{1 / 2}$ and $18.6 \mathrm{~s}^{1 / 2}$ respectively and the corresponding path of propagation is $L=2.63$ and 2.67 respectively. The zero frequency dispersion of whistlers B1, B2 and $\mathrm{B} 3$ of triplets is $81.9,95.2$ and $100.2 \mathrm{~s}^{1 / 2}$ respectively and corresponding $L$-values are $4.35,4.39$ and 4.43 respectively. This shows that the daytime whistler doublets and triplets recorded at Jammu belong to mid-latitudes. From table 1, we note that dispersion value for the doublet lies between 16 and $19 \mathrm{~s}^{1 / 2}$ which corresponds to $L$-value 1.4 . However, the computed $L$-values are 2.63 and 2.67. This shows the discrepancy between dispersion of whistler doublets and the corresponding 
derived $L$-value. The discrepancy between dispersion of whistler wave and the corresponding derived $L$-value shows that the propagation path may not be along the field line. For the reported whistlers $L>2.5$ implies that some of the waves may have propagated along higher $L$-values and after exiting from the duct, they penetrated the ionosphere and are trapped in the Earth-ionosphere wave guide. The wave-normal at the entrance into the wave guide is such that they propagated towards the equator and are received at our ground station Jammu (Singh 1993; Singh et al 1992, 2004a, 2006). In the case of triplets, the $L$-value for the path of propagation and estimated dispersion match reasonably well. The $L$-value in all cases is greater than 1.2, which corresponds to the ground station Jammu. This implies that these waves may have propagated along higher $L$-values and after exiting from the duct, they penetrated the ionosphere and propagated towards the equator. If the wave propagates in the waveguide through the process of multiple internal reflections, then there should be cut-off frequency depending upon the mode number. Such a cut-off frequency for the Earthionosphere waveguide is about $1.7 \mathrm{kHz}$ for the fundamental mode. The dynamic spectra extend well below the cut-off frequency in the present case. We have observed a number of cases in which the analysis yielded higher $L$-values but signal did not show cut-off frequency. This is probable if the wave propagates as surface wave along the Earth surface because at low frequencies Earth behaves as a good conductor. When the waves enter into the Earth-ionosphere waveguide at certain angles to the Earth's surface, it propagates through multiple reflections and various wave modes depending on wave-mode numbers and cut-off frequencies of the waveguide that also exist. If the wave normal is parallel to the surface of the waveguide, then the propagation is in surface mode and hence no cut-off frequency is observed and the present case may follow this probability. Japanese workers have reported a large number of whistlers during daytime and interpreted their propagation to the ground in terms of ducted propagation in the presence of equatorial anomaly (Tanaka and Hayakawa 1985). However, they have not reported any events of the daytime whistler doublets and triplets. The propagation of the reported doublets and triplets in the present paper cannot be considered in terms of equatorial anomaly because the estimated $L$-values are too large to apply the effect of equatorial anomaly on the propagation of these VLF waves. Therefore, we propose that the doublets and triplets observed at Jammu may have propagated in the surface mode after exiting from the duct.
From the multi-station ground-based observations of whistlers during daytime in Japan, it is found that the daytime whistlers are found to appear during the very restricted hours in the late afternoon (1400-1700 h LT). Their rate of occurrence is extremely high mainly in the form of multiflash whistlers and their dispersion lies mostly in the range of $30-40 \mathrm{~s}^{1 / 2}$ (Hayakawa and Tanaka 1978; Ohta et al 1984). Further, studies of whistler propagation, based on data collected by ground observatory have indicated that whistlers with anomalous dispersion coefficient values are likely to be closely correlated with earthquakes occurring in the longitude range. Hayakawa (1999) pointed out a possible seismic influence on the propagation of magnetospheric whistlers at low altitudes on the basis of long term ground data. By statistical analysis of such data it has been found that a number of anomalous whistlers, whose dispersion value is greater than twice the typical value, exhibit a substantial increase in coincidence with earthquake occurrence in the Japanese geographic latitude sector. It is for the first time that the correlation between seismic activity and whistler occurrence has been investigated using DEMETER satellite data (Buzzi 2006). From the recent DEMETER (WHIMAP RNF whistler data) satellite it has been found that the geomagnetic disturbances with high values of geomagnetic indices, as $K_{p}$ or $A_{p}$, play an important role in the enhancement of the occurrence of whistlers and anomalous whistlers. An exceedingly high statistical correlation between the summertime anomalous whistlers and seismic activity has been found from the analysis of 11 months DEMETER RNF whistler data averaged over the whole latitude and longitude ranges (Buzzi 2006).

The variation in the lower and the upper cutoff frequencies of the whistler doublets observed by low altitude satellite was explained in terms of the difference in $L$-value followed by the two components. It was suggested that the second component propagated at a higher $L$-value than the first one, i.e., the difference in the $L$-value between satellite position and the duct was higher for the second component, which resulted in a lower upper cut-off frequency (Strangeways 1986). The upper cut-off frequency might also result from high-altitude leakage from wide ducts as observed by Angerami (1970) on OGO3 satellite. The daytime propagation of VLF waves in the ionosphere near the equator is affected by the absorption of high frequency components known as equatorial erosion (Scarabucci 1969). Singh et al (1997) used it to explain the frequency spectrum of whistler triplets observed at Agra during night hours. This mechanism could also be used to explain the 
upper cut-off frequency of whistlers in doublets. The observed lower and upper cut-off frequencies of whistler doublets and triplets may also be explained using source characteristics such as the distribution of radiated power as a function of frequency during lightning discharge. In the absence of any measurements, this remains a speculative argument.

\section{Conclusion}

The observations of whistler doublet and triplet during daytime are new results and have not been reported earlier from any of the ground-based stations at low latitudes. The simultaneous observations of such whistlers at the ground stations and on-board satellites will be useful in acquiring further knowledge about VLF wave propagation mechanism in the inner magnetosphere and the nature of plasma structure existing at low latitudes.

\section{Acknowledgements}

This work was partly supported by DST, New Delhi under SERC project (R P S, A K S, Lalmani). K K Singh is thankful to the Council of Scientific and Industrial Research, Govt. of India, New Delhi for awarding Research Associateship under No. ACK.9/13/105/2007 EMR-1. R P Patel is thankful to the Department of Science and Technology, Govt. of India, New Delhi for awarding Research Project under SERC-Fast Track Scheme for young scientists (SR/FTP/PS12/2006). Lalmani and B L Koul are thankful to Prof. R K Wanchoo, Director, National Institute of Technology, Srinagar, Kashmir for his constant encouragement and support.

\section{References}

Angerami J A 1970 Whistler duct properties deduced from VLF observation made with the OGO 3 satellite near the magnetic equator; J. Geophys. Res. $\mathbf{7 5}$ 6115-6135.

Bernard L C 1973 A new nose extension method for whistlers; J. Atmos. Terres. Phys. 35 871-880.

Buzzi A 2006 DEMETER satellite data analysis of seismoelectromagnetic signals, Ph.D. Thesis, Department of Physics, ROMA TRE UNIV.-LPCE/CNRS Orleans University, Co-Tutorship Agreement "VINCI" Programme of Italian French University.

Chum J, Jiricek F, Santolik O, Parrot M, Diendorfer G and Fiser J 2006 Assigning the causative lightning to the whistlers observed on satellites; Annales Geophysicae 24 2921-2929.
Ferencz O E, Ferencz Cs, Steinbach P, Lichtenberger J, Hamar D, Parrot M, Lefeuvre F and Berthelier J J 2007 The effect of subionospheric propagation on whistlers recorded by the DEMETER satellite observation and modeling; Annales Geophysicae 25 1103-1112.

Hamar D, Tarcsai G, Lichtenberger J, Smith A J, Yearby K H 1990 Fine structures of whistlers recorded digitally at Hally, Antarctica; J. Atmos. Terres. Phys. 52 801-810.

Hayakawa M 1999 Atmospheric and Ionospheric Electromagnetic Phenomena Related to Earthquake Predictions, Terra.SCI.PUB.CO. Tokyo, Japan.

Hayakawa M and Tanaka Y 1978 On the propagation of low latitude whistlers; Rev. Geophys. Space Phys. 16 $111-123$.

Helliwell R A 1965 Whistlers and related ionospheric phenomena (Stanford: Stanford University Press).

Lichtenberger J, Tarcsai G, Pasztor S, Ferenz Cs, Hamar D, Molchanov O A, Golyavin A M 1991 Whistler doublets and hyperfine structures recorded digitally by the signal analyzer and sampler on the active satellite; J. Geophys. Res. 96 21,149-21,158.

Ohta K, Hayakawa M and Tanaka Y 1984 Ducted propagation of daytime whistlers at low latitudes as deduced from ground-based direction findings; J. Geophys. Res. $897557-7564$.

Park C G 1972 Methods of determining electron concentration in the magnetosphere from nose whistlers; Tech. Report No. 3454-1, Radio Science Lab. (Stanford: Stanford University).

Scarabucci R R 1969 Interpretation of VLF signals observed on the OGO 4 satellite. Tech. Rep. 3418-2, Stanford Electronic Lab., Stanford, California.

Singh R P 1993 Whistler studies at low latitudes: a review; Indian Journal of Radio and Space Physics 22 139-155.

Singh U P, Singh A K, Lalmani Singh R P and Singh R N 1992 Hybrid-mode propagation of whistlers at low latitudes; Indian Journal of Radio and Space Physics 21 246-249.

Singh B, Singh R and Singh R 1997 Whistler triplets, bands and fine structures observed in a low latitude ground station; Geophys. Res. Lett. 24 2507-2510.

Singh R P, Singh A K and Singh D K 1998 Plasmaspheric parameters as determined from whistler spectrograms: a review; J. Atmos. Terres. Phys. 60 494-508.

Singh R P, Singh D K, Singh A K, Hamar D and Lichtenberger J 1999 Application of matched filtering and parameter estimation technique to low latitude whistlers; J. Atmos. Solar-Terres. Phys. 61 1081-1092.

Singh R, Patel R P, Singh R P and Lalmani 2000 An experimental study of hiss triggered chorus emissions at low latitudes; Earth Planet and Space 52 37-40.

Singh B and Hayakawa M 2001 Propagation modes of lowand very-low-latitude whistlers; J. Atmos. Solar-Terres. Phys. 63 1133-1147.

Singh R P, Singh R, Lalmani, Hamar D and Lichtenberger J 2004a Application of matched filtering to short whistlers recorded at low latitudes; J. Atmos. Solar-Terres. Phys. 66 407-413.

Singh K K, Singh R, Singh R P and Shyampati 2004b Hisslers: Quasi-periodic VLF noise forms observed at low latitude ground station Jammu $(L=1.17)$; Geophys. Res. Lett. 31 L19802, doi:10.1029/2004 GL 020468.

Singh R P, Singh K, Ashok K Singh, Hamar D and Lichtenberger J 2006 Matched filtering analysis of diffused whistlers and its propagation characteristics at low latitudes; J. Atmos. Solar-Terres. Phys. 68 710-714.

Singh K, Singh R, Singh A K and Singh R P 2007 Propagation characteristics and generation mechanism of 
ELF/VLF Hiss observed at low latitude, Jammu; Earth Moon Planets 100 17-29.

Somayajulu V V, Rao M and Tantry B A P 1972 Whistlers at low latitudes; Indian Journal of Radio and Space Physics 1 102-118.

Strangeways H J 1986 Whistler leakage from narrow ducts; J. Atmos. Terres. Phys. 48 455-463.
Tanaka Y and Hayakawa M 1985 On the propagation of daytime whistlers at low latitudes; J. Geophys. Res. 90 3457-3464.

Tarcsai G 1975 Routine whistler analysis by means of accurate curve fitting; J. Atmos. Terres. Phys. 37 1447-1457.

Thomson R J 1977 PL whistlers; Planetary Space Science 25 1037-1043.

MS received 6 August 2007; revised 2 February 2008; accepted 19 February 2008 